\title{
Direct Localized Boundary-Domain Integro-Differential Formulations for Physically Nonlinear Elasticity of Inhomogeneous Body
}

\author{
S.E. Mikhailov \\ Div. of Mathematics, Glasgow Caledonian University, \\ Glasgow, G4 0BA, UK, s.mikhailov@gcal.ac.uk
}

Keywords: Non-linear Elasticity, Variable Coefficients, Direct Formulation, Integro-Differential Equation, Localization, Mesh-based Discretization, Mesh-less Discretization

\begin{abstract}
A static mixed boundary value problem of physically nonlinear elasticity for a continuously inhomogeneous body is considered. Using the two-operator Green-Betti formula and the fundamental solution of an auxiliary linear operator, a non-standard boundary-domain integro-differential formulation of the problem is presented, with respect to the displacements and their gradients. Using a cut-off function approach, the corresponding localized parametrix is constructed to reduce the nonlinear boundary value problem to a nonlinear localized boundary-domain integro-differential equation. Algorithms of mesh-based and mesh-less discretizations are presented resulting in sparsely populated systems of nonlinear algebraic equations.
\end{abstract}

\section{Introduction}

Application of the Boundary Integral Equation (BIE) method (boundary element method, elastic potential method) to linear elasticity problems for homogeneous bodies has been intensively developed over recent decades. Using fundamental solutions of auxiliary linear elastic problems (with the initial elastic coefficients), the non-linearly elastic or elasto-plastic problems for homogeneous material also can be reduced to non-linear boundary-domain integral equations with hyper-singular integrals, see e.g. [1-4]. However, the fundamental solution is usually highly non-local, which leads after discretization to a system of algebraic equations with a dense matrix. Moreover, the fundamental solution is generally not available in an explicit form if the coefficients of the auxiliary problem vary in space, i.e. if the material is inhomogeneous (functionally graded).

To prevent such difficulties, some parametrices localized by cut-off function multiplication were constructed and implemented in [5] for linear scalar (heat transfer) equation in inhomogeneous medium. This reduced the linear Boundary Value Problem (BVP) with variable coefficient to a linear Localized 
Boundary-Domain Integral or Integro-Differential Equation (LBDIE or LBDIDE), which leaded after a mesh-based or mesh-less discretization to a linear algebraic system with a sparse matrix. Some numerical implementations of the linear LBDIE were presented in $[6,7]$, while slightly different LBDI(D)Es were employed in $[8,9]$.

Another approach based on local parametrices that are Green functions for an auxiliary problem on local spherical domains, was used in [10-12] to reduce some linear and nonlinear scalar problems with variable coefficients, and in [13] a linear elasticity problem for a body with a special inhomogeneity, to a local boundary-domain integral equations. Note also that the Green function of the plane Laplace equation was used in [14] as a parametrix for the axially symmetric problem of heat transfer with variable coefficients.

Extending approach of [5], the mixed BVP for a second order scalar nonlinear (quasi-linear) elliptic PDE with the variable coefficient dependent on the unknown solution was reduced in $[15,17]$ to quasilinear LBDIDEs. When the variable coefficient depends also on the BVP solution gradient, some quasi-linear two-operator LBDIDEs were obtained in $[16,17]$.

In this paper, we extend the approach of $[5,16,17]$ to the mixed BVP for the system of quasi-linear partial differential equations of physically nonlinear elasticity (with small deformation gradients) for continuously inhomogeneous body. First, we reduce the BVP to a direct two-operator nonlinear BDIDE of the second kind. The equation includes at most first derivatives of the unknown solution, weakly singular integrals over the domain and at most Cauchy-type singular integrals over the boundary. Then we present a localized version of the BDIDE and describe its mesh-based and mesh-less discretizations. A short description of the method was presented in [18], while its formulation for inhomogeneous elastoplasticity was given in [19].

\section{Nonlinear Elasticity Problem, Two-operator Green-Betti Identity and BDIDE}

Let us consider an inhomogeneous material, occupying an $n$-dimensional domain $\Omega \in \mathbb{R}^{n}$, where $n=2$ or $n=3$. Its physically-nonlinear elastic constitutive relations (presuming small displacement gradients) can be written in the form

$$
\sigma_{i j}(\nabla u(x), u(x), x)=a_{i j k l}(\nabla u(x), u(x), x) \frac{\partial u_{k}(x)}{\partial x_{l}},
$$

where $\sigma=\sigma_{i j}$ is the stress tensor, $u(x)=u_{i}(x)$ is the displacement vector; the tensor $a=a_{i j k l}(\nabla u, u, x)$ is a known function of $u(x)$ and of its gradient $\nabla u(x)=u_{i, j}$. The comma in front of a superscript means derivative in the corresponding coordinate, and summation in repeated indices is supposed from 1 to $n$ unless stated otherwise. The dependence of $a$ and $\sigma$ on the displacement $u$ (in addition 
to the dependence on $\nabla u$ ) is left here for generality.

Note that a dependence $\sigma_{i j}=\sigma_{i j}(\nabla u(x), u(x), x)$, such that $\sigma_{i j}(0, u(x), x)=0$ can always be presented in form (1), since the mean value theorem implies,

$$
\sigma_{i j}(\nabla u(x), u(x), x)=\frac{\partial \sigma_{i j}(g, u(x), x)}{\partial g_{k l}} u_{k, l}(x), \quad \text { where } g=t \nabla u(x)
$$

for some $t$ in the segment $[0,1]$.

Substituting (1) in the equilibrium equations

$$
\frac{\partial \sigma_{i j}(\nabla u(x), u(x), x)}{\partial x_{j}}=f_{i}(x), \quad i=1, \ldots, n,
$$

and taking into account boundary conditions, we arrive at the following mixed boundary-value problem of physically nonlinear elasticity in a bounded domain $\Omega$ for the unknown displacement vector $u$,

$$
\begin{aligned}
{\left[L_{i k}(u) u_{k}\right](x):=\frac{\partial}{\partial x_{j}}\left[a_{i j k l}(\nabla u(x), u(x), x) \frac{\partial u_{k}(x)}{\partial x_{l}}\right]=f_{i}(x), } & x \in \Omega, \\
u_{i}(x) & =\check{u}_{i}(x), \quad x \in \partial_{D} \Omega, \\
{\left[T_{i k}(u) u_{k}\right](x):=a_{i j k l}(\nabla u(x), u(x), x) \frac{\partial u_{k}(x)}{\partial x_{l}} n_{j}(x)=\check{t}_{i}(x), } & x \in \partial_{N} \Omega .
\end{aligned}
$$

Here $a_{i j k l}=a_{j i k l}=a_{i j l k}=a_{k l i j} ; f_{i}(x)$ is a known volume force vector (taken with the opposite sign); $n_{i}(x)$ is an outward normal vector to the boundary $\partial \Omega ;[T(u) u](x)=\left[T_{i k}(u) u_{k}\right](x)$ is the traction vector at a boundary point $x$, while $T(u)=T_{i k}(u)$ is the traction differential operator; $\check{u}(x)$ and $\check{t}(x)$ are known displacements and tractions on the parts $\partial_{D} \Omega$ and $\partial_{N} \Omega$ of the boundary, respectively.

Let us fix a point $y$ and consider the following auxiliary differential operators of the linear elasticity with constant (frozen) coefficients,

$$
\begin{gathered}
{\left[L_{i k}^{(y)}(u) v_{k}\right](x):=\frac{\partial}{\partial x_{j}}\left[a_{i j k l}(\nabla u(y), u(y), y) \frac{\partial v_{k}(x)}{\partial x_{l}}\right]} \\
{\left[T_{i k}^{(y)}(u) v_{k}\right](x):=a_{i j k l}(\nabla u(y), u(y), y) \frac{\partial v_{k}(x)}{\partial x_{l}} n_{j}(x) .}
\end{gathered}
$$

Integrating by parts, we have the first Green identities for the differential operators

$$
\begin{aligned}
& {[L(u) u](x)=\left[L_{i k}(u) u_{k}\right](x) \text { and }\left[L^{(y)}(u) v\right](x)=\left[L_{i k}^{(y)}(u) v_{k}\right](x),} \\
& \int_{\Omega} v_{i}(x)\left[L_{i k}(u) u_{k}\right](x) d \Omega(x)= \\
& \int_{\partial \Omega} v_{i}(x)\left[T_{i k}(u) u_{k}\right](x) d \Gamma(x)-\int_{\Omega} \frac{\partial v_{i}(x)}{\partial x_{j}} a_{i j k l}(\nabla u(x), u(x), x) \frac{\partial u_{k}(x)}{\partial x_{l}} d \Omega(x), \\
& \int_{\Omega} u_{i}(x)\left[L_{i k}^{(y)}(u) v_{k}\right](x) d \Omega(x)= \\
& \int_{\partial \Omega} u_{i}(x)\left[T_{i k}^{(y)}(u) v_{k}\right](x) d \Gamma(x)-\int_{\Omega} \frac{\partial u_{i}(x)}{\partial x_{j}} a_{i j k l}(\nabla u(y), u(y), y) \frac{\partial v_{k}(x)}{\partial x_{l}} d \Omega(x),
\end{aligned}
$$

where $u(x)$ and $v(x)$ are arbitrary vector-functions for that the operators and integrals in the above expressions have sense. Subtracting the identities from each other and taking into account the symmetry 
of the tensor $a_{i j k l}$, we derive the two-operator second Green-Betti identity,

$$
\begin{aligned}
& \int_{\Omega}\left\{u(x)\left[L^{(y)}(u) v\right](x)-v(x)[L(u) u](x)\right\} d \Omega(x)= \\
& \int_{\partial \Omega}\left\{u(x)\left[T^{(y)}(u) v\right](x)-v(x)[T(u) u](x)\right\} d \Gamma(x)+\int_{\Omega}[\nabla v(x)] \tilde{a}(u ; x, y) \nabla u(x) d \Omega(x), \\
& \tilde{a}(u ; x, y)=\tilde{a}_{i j k l}(u ; x, y):=\left[a_{i j k l}(\nabla u(x), u(x), x)-a_{i j k l}(\nabla u(y), u(y), y)\right] .
\end{aligned}
$$

Note that if $L(u)=L^{(y)}(u)$, i.e. $L(u)$ is a linear operator with constant coefficients, then the last domain integral disappears in eq (7), which thus degenerates into the classical second Green-Betti identity.

For a fixed $u$ and $y$, let $F^{(y)}(u ; x, y)=F_{k m}^{(y)}(u(y), \nabla u(y), x, y)$ be a fundamental solution for the linear differential operator $\left[L_{i k}^{(y)}(u) v_{k}\right](x)$ with constant (with respect to $x$ ) coefficients, i.e.,

$$
\left[L_{i k}^{(y)}(u) F_{k m}^{(y)}(u ; \cdot, y)\right](x) \quad:=a_{i j k l}(\nabla u(y), u(y), y) \frac{\partial^{2} F_{k m}^{(y)}(u(y), \nabla u(y), x, y)}{\partial x_{j} \partial x_{l}}=\delta_{i m} \delta(x-y),
$$

where $\delta_{i m}$ is the Kronecker symbol and $\delta(x-y)$ is the Dirac delta-function. Note that generally $F^{(y)}(u ; x, y)$ is not a parametrix for the original operator $L(u)$ if the tensor $a$ depends on $\nabla u$.

If the material is isotropic, then

$$
\begin{gathered}
a_{i j k l}(\nabla u(y), u(y), y)=\lambda(\nabla u(y), u(y), y) \delta_{i j} \delta_{k l}+\mu(\nabla u(y), u(y), y)\left(\delta_{i k} \delta_{j l}+\delta_{i l} \delta_{j k}\right), \\
\mu(\nabla u(y), u(y), y)>C>0, \quad \lambda(\nabla u(y), u(y), y)+\frac{2}{3} \mu(\nabla u(y), u(y), y)>C>0 .
\end{gathered}
$$

In this case, $F_{i m}^{(y)}(u ; x, y)$ is the Kelvin-Somigliana solution,

$$
F_{i m}^{(y)}(u ; x, y)=\frac{-1}{4 \pi}\left\{\frac{-\delta_{i m} \ln r-r_{, i} r_{, m}}{\lambda(\nabla u(y), u(y), y)+2 \mu(\nabla u(y), u(y), y)}+\frac{-\delta_{i m} \ln r+r_{, i} r_{, m}}{\mu(\nabla u(y), u(y), y)}\right\}
$$

for the plane strain state; for the plane stress, $\lambda$ in (9) and (10) should be replaced by $\lambda^{*}=2 \lambda \mu /(\lambda+2 \mu)$. In the $3 \mathrm{D}$ case,

$$
F_{i m}^{(y)}(u ; x, y)=\frac{-1}{8 \pi r}\left\{\frac{\delta_{i m}-r_{, i} r_{, m}}{\lambda(\nabla u(y), u(y), y)+2 \mu(\nabla u(y), u(y), y)}+\frac{\delta_{i m}+r_{, i} r_{, m}}{\mu(\nabla u(y), u(y), y)}\right\}
$$

Here $r:=\sqrt{\left(x_{i}-y_{i}\right)\left(x_{i}-y_{i}\right)}, \quad r_{, i}:=\partial r / \partial x_{i}=\left(x_{i}-y_{i}\right) / r$. For anisotropic material, the fundamental solution can be written down in an analytical form for arbitrary anisotropy in the 2D case and for some particular anisotropy in the 3D case; otherwise, it can be expressed as a linear integral over a circle $[20-22]$.

Assuming $u(x)$ is a solution of nonlinear system (2) and using the fundamental solution $F^{(y)}(u ; x, y)$ as $v(x)$ in the Green identity (7) similar to the linear case, c.f. [5,23], we obtain the following non-linear two-operator third Green identity,

$$
\begin{array}{r}
c(y) u(y)-\int_{\partial \Omega} u(x)\left[T^{(y)} F^{(y)}(u ; \cdot, y)\right](x) d \Gamma(x)+\int_{\partial \Omega} F^{(y)}(u ; x, y)[T(u) u](x) d \Gamma(x)- \\
\int_{\Omega}\left[\nabla^{(x)} F^{(y)}(u ; x, y)\right] \tilde{a}(u ; x, y) \nabla u(x) d \Omega(x)=\int_{\Omega} F^{(y)}(u ; x, y) f(x) d \Omega(x),
\end{array}
$$


where $c$ is a tensor, $c_{i j}$, such that $c_{i m}(y)=\delta_{i m}$ if $y \in \Omega ; c_{i m}(y)=0$ if $y \notin \bar{\Omega} ; c_{i m}(y)=\frac{1}{2} \delta_{i m}$ if $y$ is a smooth point of the boundary $\partial \Omega$; and $c_{i m}(y)=c_{i m}(a(y), \alpha(y))$ is a function of the anisotropy tensor $a(y)$ and the interior space angle $\alpha(y)$ at a corner point $y$ of the boundary $\partial \Omega$.

Substituting boundary conditions (3), (4) into eq (12) and using it at $y \in \bar{\Omega}$, we arrive at a (united) nonlinear two-operator BDIDE for $u(x)$ at $x \in \bar{\Omega}$

$$
\begin{gathered}
c(y) u(y)-\int_{\partial_{N} \Omega} u(x)\left[T^{(y)}(u) F^{(y)}(u ; \cdot, y)\right](x) d \Gamma(x)+\int_{\partial_{D} \Omega} F^{(y)}(u ; x, y)[T(u) u](x) d \Gamma(x)- \\
\int_{\Omega}\left[\nabla^{(x)} F^{(y)}(u ; x, y)\right] \tilde{a}(u ; x, y) \nabla u(x) d \Omega(x)=\mathcal{F}(y), \quad y \in \bar{\Omega}, \quad(13) \\
\mathcal{F}(y):=\int_{\partial_{D} \Omega} \check{u}(x)\left[T^{(y)}(u) F^{(y)}(u ; \cdot, y)\right](x) d \Gamma(x)-\int_{\partial_{N} \Omega} F^{(y)}(u ; x, y) \check{t}(x) d \Gamma(x)+ \\
\int_{\Omega} F^{(y)}(u ; x, y) f(x) d \Omega(x) .
\end{gathered}
$$

BDIDE (13) is the second kind equation, which includes at most the first derivatives of the unknown solution $u(x)$, both directly in the domain integral term in the left hand side and through the coefficient $a(\nabla u, u, \cdot)$ in the operators $T(u), T^{(y)}(u)$ and the functions $F^{(y)}(u ; x, y)$ and $\tilde{a}(u ; x, y)$. The function $\left[\nabla^{(x)} F^{(y)}(u ; x, y)\right]$ is at most weakly singular in $\Omega$, and taking into account that $\tilde{a}(u ; x, y) \rightarrow 0$ as $x \rightarrow y$, we obtain that the domain integral is a smoothing operator with respect to $u$, for (sufficiently) smooth functions $a$ and $u$. The boundary integrals have at most the Cauchy-type singularity.

Some other (e.g. segregated) BDIDEs can be obtained if one substitutes $\check{u}(x)$ for $u(x)$ also in the out-of-integral term of $(13)$ at $y \in \partial_{D} \Omega$, considers the unknown boundary displacements $u$ on $\partial_{N} \Omega$ and/or tractions $T(u) u$ on $\partial_{D} \Omega$ as new variables formally segregated from $u$ in $\Omega$, or applies the boundary traction operator to (13).

BDIDE (13) can be reduced after some discretization to a system of nonlinear algebraic equation and solved numerically. The system will include unknowns not only on the boundary but also at internal points. Moreover, since the fundamental solutions, c.f. (10), (11), are highly non-local, the matrix of the system will be fully populated and this makes its numerical solution more expensive. To avoid this difficulty, we present below some ideas of constructing localized parametrices and consequently Localized BDIDEs (LBDIDEs).

\section{Localized Parametrix and LBDIDE}

Let $\chi(x, y)$ be a cut-off function, such that $\chi(y, y)=1$ and $\chi(x, y)=0$ at $x$ not belonging to closure of an open localization domain $\omega(y)$ (a vicinity of $y$ ), see Fig.1, and let $P_{\omega}^{(y)}(u ; x, y)=\chi(x, y) F^{(y)}(u ; x, y)$. The simplest example is

$$
\chi(x, y)=\left\{\begin{array}{ll}
1, & x \in \bar{\omega} \\
0, & x \notin \bar{\omega}
\end{array} \Rightarrow P_{\omega}^{(y)}(u ; x, y)= \begin{cases}F^{(y)}(u ; x, y), & x \in \bar{\omega}(y) \\
0, & x \notin \bar{\omega}(y)\end{cases}\right.
$$


Other examples of the cut-off functions having different smoothness are presented in $[5,6,17]$ for some shapes of $\omega$.

Then $P_{\omega}^{(y)}(u ; x, y)$ is a localized parametrix of the linear operator $L^{(y)}$, i.e.,

$$
L_{i k}^{(y)}(u) P_{k m \omega}^{(y)}(u ; x, y)=\delta_{i m} \delta(x-y)+R_{i m \omega}^{(y)}(u ; x, y),
$$

where the remainder

$$
R_{i m \omega}^{(y)}=-L_{i k}^{(y)}\left((1-\chi) F_{k m}^{(y)}\right)=a_{i j k l}(\nabla u(y), u(y), y)\left[F_{k m}^{(y)} \frac{\partial^{2} \chi}{\partial x_{j} \partial x_{l}}+\frac{\partial F_{k m}^{(y)}}{\partial x_{j}} \frac{\partial \chi}{\partial x_{l}}+\frac{\partial F_{k m}^{(y)}}{\partial x_{l}} \frac{\partial \chi}{\partial x_{j}}\right]
$$

is at most weakly singular at $x=y$, at least if $\chi$ is smooth enough on $\bar{\omega}(y)$. The parametrix $P_{\omega}^{(y)}(u ; x, y)$ has the same singularity as $F^{(y)}(x, y)$ at $x=y$. Both $P_{\omega}^{(y)}(u ; x, y)$ and $R_{\omega}^{(y)}(u ; x, y)$ are localized (nonzero) only on $\bar{\omega}(y)$.

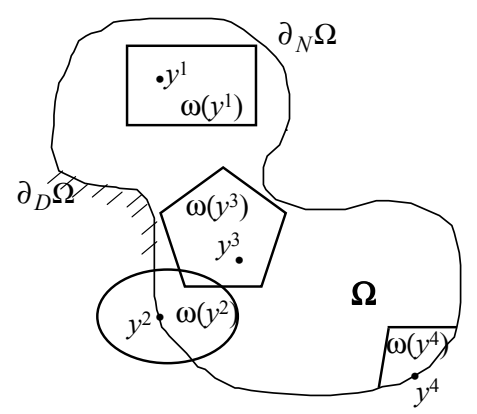

Figure 1: Body $\Omega$ with localization domains $\omega\left(y^{i}\right)$

Suppose $\chi(x, y)$ is smooth in $x \in \bar{\omega}(y)$ but not necessarily zero at $x \in \partial \omega(y)$. Then $P_{\omega}^{(y)}(u ; x, y)$ is a discontinuous localized parametrix at $x \in \mathbb{R}^{n}$ and $P_{\omega}^{(y)}(u ; x, y)=R_{\omega}^{(y)}(u ; x, y)=0$ if $x \notin \bar{\omega}(y)$. Substituting $P_{\omega}^{(y)}(u ; x, y)$ for $v(x)$ in eq $(7)$ and replacing $\Omega$ by the intersection $\omega(y) \cap \Omega$, we arrive at the localized parametrix-based two-operator third Green identity on $\bar{\omega}(y) \cap \bar{\Omega}$,

$$
\begin{gathered}
c_{\omega}(y) u(y)-\int_{\bar{\omega}(y) \cap \partial \Omega}\left\{u(x)\left[T^{(y)}(u) P_{\omega}^{(y)}(u ; \cdot, y)\right](x)-P_{\omega}^{(y)}(u ; x, y)[T(u) u](x)\right\} d \Gamma(x)- \\
\int_{\Omega \cap \partial \omega(y)}\left\{u(x)\left[T^{(y)}(u) P_{\omega}^{(y)}(u ; \cdot, y)\right](x)-P_{\omega}^{(y)}(u ; x, y)[T(u) u](x)\right\} d \Gamma(x)- \\
\int_{\omega(y) \cap \Omega}\left[\nabla^{(x)} P_{\omega}^{(y)}(u ; x, y)\right] \tilde{a}(u ; x, y) \nabla u(x) d \Omega(x)+\int_{\omega(y) \cap \Omega} R_{\omega}^{(y)}(u ; x, y) u(x) d \Omega(x)= \\
\int_{\omega(y) \cap \Omega} P_{\omega}^{(y)}(u ; x, y) f(x) d \Omega(x) .
\end{gathered}
$$

The last integral in the left hand side of (15) disappears if $\chi(x, y)$ is given by (14). If the point $y$ is situated inside the localization domain $\omega(y)$ or on the intersection of the local and global boundaries, $\partial \omega(y) \cap \Omega$, the coefficient $c_{\omega}(y)$ in (15) is the same as $c(y)$ in equation (12). However, if $y$ is situated 
inside the global domain $\Omega$ but on the boundary of localization domain $\omega(y)$, then $c_{\omega i m}(y)=\frac{1}{2} \delta_{i m}$ if $y$ is a smooth point of the boundary $\partial \omega$; and $c_{\omega i m}(y)=c_{\omega i m}\left(a(y), \alpha_{\omega}(y)\right)$ is a function of the anisotropy tensor $a(y)$ and the interior space angle $\alpha_{\omega}(y)$ at a corner point $y$ of the boundary $\partial \omega$.

Substituting boundary conditions (3) and (4) into the integral terms of eq (15) and employing it at $y \in \bar{\Omega}$, we arrive at the united formulation of nonlinear two-operator Localized Boundary-Domain Integro-Differential Equation (LBDIDE) of the second kind, for $u(x), x \in \bar{\Omega}$,

$$
\begin{gathered}
c_{\omega}(y) u(y)-\int_{\bar{\omega}(y) \cap \partial_{N} \Omega} u(x)\left[T^{(y)}(u) P_{\omega}^{(y)}(u ; \cdot, y)\right](x) d \Gamma(x)+\int_{\bar{\omega}(y) \cap \partial_{D} \Omega} P_{\omega}^{(y)}(u ; x, y)[T(u) u](x) d \Gamma(x)- \\
\int_{\Omega \cap \partial \omega(y)}\left\{u(x)\left[T^{(y)}(u) P_{\omega}^{(y)}(u ; \cdot, y)\right](x)-P_{\omega}^{(y)}(u ; x, y)[T(u) u](x)\right\} d \Gamma(x)- \\
\int_{\omega(y) \cap \Omega}\left\{\left[\nabla^{(x)} P_{\omega}^{(y)}(u ; x, y)\right] \tilde{a}(u ; x, y) \nabla u(x)-R_{\omega}^{(y)}(u ; x, y) u(x)\right\} d \Omega(x)=\mathcal{F}_{\omega}(u ; y), y \in \bar{\Omega}, \quad(1 \\
\mathcal{F}_{\omega}(u ; y):=\int_{\bar{\omega}(y) \cap \partial_{D} \Omega} \check{u}(x)\left[T^{(y)}(u) P_{\omega}^{(y)}(u ; \cdot, y)\right](x) d \Gamma(x)- \\
\int_{\bar{\omega}(y) \cap \partial_{N} \Omega} P_{\omega}^{(y)}(u ; x, y) \check{t}(x) d \Gamma(x)+\int_{\omega(y) \cap \Omega} P_{\omega}^{(y)}(u ; x, y) f(x) d \Omega(x) .
\end{gathered}
$$

If a cut-off function $\chi(x, y)$ vanishes at $x \in \partial \omega(y)$ with vanishing normal derivatives, then the integral along $\Omega \cap \partial \omega(y)$ disappears in eq (16).

\section{Discretization of Nonlinear Two-operator LBDIE}

To reduce quasi-linear LBDIDE (16) to a sparsely populated system of quasi-linear algebraic equations e.g. by the collocation method, one has to employ a local interpolation or approximation formula for the unknown function $u(x)$, for example associated with a mesh-based or mesh-less discretization.

\subsection{Mesh-based discretization.}

Suppose the domain $\Omega$ is covered by a mesh of closures of disjoint domain elements $e_{k}$ with nodes set up at the corners, edges, faces, or inside the elements. Let $J$ be the total number of nodes $x^{i}(i=1,2, \ldots, J)$. One can use each node $x^{i}$ as a collocation point for the LBDIDE with a localization domain $\omega\left(x^{i}\right)$. Let the part of $\omega\left(x^{i}\right)$ covered by an element $e_{k}$ is denoted by $\omega^{i k}=\omega\left(x^{i}\right) \cap e_{k}$

Let the union of closures of the domain elements that intersect with $\omega\left(x^{i}\right)$ be called the total localization domain $\tilde{\omega}^{i}$, Fig. 2. Evidently the closure $\bar{\omega}\left(x^{i}\right) \cap \bar{\Omega}$ belongs to $\tilde{\omega}^{i}$. If $\omega\left(x^{i}\right)$ is sufficiently small, then $\tilde{\omega}^{i}$ consists only of the elements adjacent to the collocation point $x^{i}$. If $\omega\left(x^{i}\right)$ is ab initio chosen as consisting only of the elements adjacent to the collocation point $x^{i}$, then $\tilde{\omega}^{i}=\bar{\omega}\left(x^{i}\right)$. Let $u\left\{\tilde{\omega}^{i}\right\}$ be the array of the function values $u\left(x^{j}\right)$ at the node points $x^{j} \in \tilde{\omega}^{i}$ and $J_{\tilde{\omega}^{i}}$ be the number of those node points. 


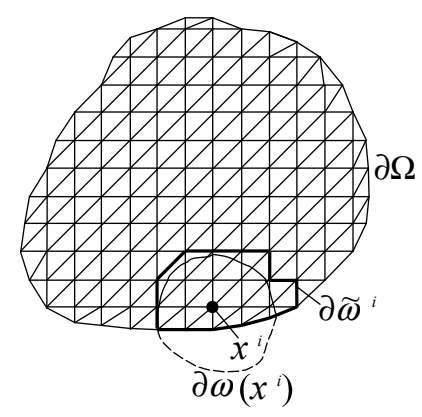

Figure 2: Localization domain $\omega\left(x^{i}\right)$ and a total localization domain $\tilde{\omega}^{i}$ associated with a collocation point $x^{i}$ of a body $\Omega$ for mesh-based discretizations

Let

$$
u(x)=\sum_{j} u\left(x^{j}\right) \phi_{k j}(x)
$$

be a continuous piece-wise smooth interpolation of $u(x)$ at any point $x \in \Omega$ along the values $u\left(x^{j}\right)$ at the node points $x^{j}$ belonging to the same element $\bar{e}_{k} \subset \Omega$ as $x$, and the shape functions $\phi_{k j}(x)$ be localized on $\bar{e}_{k}$. Collecting the interpolation formulae, we have for any $x \in \tilde{\omega}^{i}$,

$$
\begin{aligned}
u(x) & =\sum_{x^{j} \in \tilde{\omega}^{i}} u\left(x^{j}\right) \Phi_{j}(x), & \Phi_{j}(x) & = \begin{cases}\phi_{k j}(x) & \text { if } x, x^{j} \in \bar{e}_{k} \\
0 & \text { otherwise }\end{cases} \\
\nabla u(x) & =\sum_{x^{j} \in \tilde{\omega}^{i}} u\left(x^{j}\right) \nabla \Phi_{j}(x), & \nabla \Phi_{j}(x) & = \begin{cases}\nabla \phi_{k j}(x) & \text { if } x, x^{j} \in \bar{e}_{k} \\
0 & \text { otherwise }\end{cases}
\end{aligned}
$$

Consequently, $\Phi_{j}(x)=\nabla \Phi_{j}(x)=0$ if $x \in \tilde{\omega}^{i}$ but $x^{j} \notin \tilde{\omega}^{i}$.

Since interpolation (18) is piece-wise smooth, expressions (19) deliver different values for $\nabla u(x)$ on the element interfaces and particularly at apexes $x^{i}$ of different adjoint elements $e_{k}$. For LBDIDE (16), one has to estimate $\nabla u(y)$ to calculate the coefficient $a(\nabla u(y), u(y), y)$ and, consequently $T^{(y)}(u)$, $P^{(y)}(u ; x, y)$ and $R^{(y)}(u ; x, y)$ at $y=x^{i}$. For this reason, one can not directly substitute interpolation (18)-(19) in LBDIDE (16) and employ the equation at the collocation points.

To circumvent this, let us consider LBDIDE (16) at a collocation point $x^{i}$ not over the whole localization domain $\omega\left(x^{i}\right)$ but over its pieces $\omega^{i k}=\omega\left(x^{i}\right) \cap e_{k}$, substitute interpolation (18)-(19), and then sum up the LBDIDEs for all $k$ with non-empty $\omega^{i k}$. The procedure is similar to the one for piecewise smooth localization considered in [5, Section 3.3]. The resulting out-of-integral coefficient $c_{\omega}\left(x^{i}\right)=$ $\sum_{k} c_{\omega^{i k}}\left(x^{i}\right)=c\left(x^{i}\right)$ will correspond to the position of $x^{i}$ in the whole localization domain $\omega\left(x^{i}\right)$ (or, the same, in $\Omega$ ) but $a\left(\nabla u\left(x^{i}\right), u\left(x^{i}\right), x^{i}\right)$ and, consequently $\tilde{a}\left(u ; x, x^{i}\right) T^{\left(x^{i}\right)}(u), P^{\left(x^{i}\right)}(u ; x, y)$ and $R^{\left(x^{i}\right)}(u ; x, y)$ will depend on the integration element $e_{k}$ and will be denoted by $a^{k}\left(\nabla u\left(x^{i}\right), u\left(x^{i}\right), x^{i}\right)$, $\tilde{a}^{k}\left(u ; x, x^{i}\right), T^{i k}(u), P^{i k}(u ; x, y)$ and $R^{i k}(u ; x, y)$, respectively.

Then we arrive at the following system of $J \times n$ quasi-linear algebraic equations for $J \times n$ unknowns 
$u_{m}\left(x^{j}\right), x^{j} \in \bar{\Omega}, m=1, \ldots, n$,

$$
c\left(x^{i}\right) u\left(x^{i}\right)+\sum_{x^{j} \in \tilde{\omega}^{i}} K_{i j}\left(u\left\{\tilde{\omega}^{i}\right\}\right) u\left(x^{j}\right)=\mathcal{F}_{\omega}\left(u\left\{\tilde{\omega}^{i}\right\} ; x^{i}\right), \quad x^{i} \in \bar{\Omega}, \quad \text { no sum in } i .
$$

For fixed indices $i, j$, the $n \times n$ tensor $K_{i j}\left(u\left\{\tilde{\omega}^{i}\right\}\right)$ is

$$
\begin{aligned}
& K_{i j}\left(u\left\{\tilde{\omega}^{i}\right\}\right)= \sum_{k: \omega^{i k} \neq \emptyset}\left[-\int_{\bar{\omega}^{i k} \cap \partial_{N} \Omega} \phi_{k j}(x)\left[T^{\left(x^{i}\right)}\left(u\left\{\tilde{\omega}^{i}\right\}\right) P_{\omega}^{\left(x^{i}\right)}\left(u\left\{\tilde{\omega}^{i}\right\} ; \cdot, x^{i}\right)\right](x) d \Gamma(x)+\right. \\
& \int_{\bar{\omega}^{i k} \cap \partial_{D} \Omega} P_{\omega}^{\left(x^{i}\right)}\left(u\left\{\tilde{\omega}^{i}\right\} ; x, x^{i}\right)\left[T\left(u\left\{\tilde{\omega}^{i}\right\}\right) \phi_{k j}\right](x) d \Gamma(x)- \\
& \int_{\Omega \cap \partial \omega^{i k}} \phi_{k j}(x)\left[T^{\left(x^{i}\right)}\left(u\left\{\tilde{\omega}^{i}\right\}\right) P_{\omega}^{\left(x^{i}\right)}\left(u\left\{\tilde{\omega}^{i}\right\} ; \cdot, x^{i}\right)\right](x) d \Gamma(x)+ \\
& \int_{\Omega \cap \partial \omega^{i k}} P_{\omega}^{\left(x^{i}\right)}\left(u\left\{\tilde{\omega}^{i}\right\} ; x, x^{i}\right)\left[T\left(u\left\{\tilde{\omega}^{i}\right\}\right) \phi_{k j}\right](x) d \Gamma(x)- \\
&\left.\int_{\omega^{i k} \cap \Omega} \nabla^{(x)} P_{\omega}^{\left(x^{i}\right)}\left(u\left\{\tilde{\omega}^{i}\right\} ; x, x^{i}\right)\right] \tilde{a}\left(u\left\{\tilde{\omega}^{i}\right\} ; x, x^{i}\right) \nabla \phi_{k j}(x) d \Omega(x)+ \\
&\left.\int_{\omega^{i k} \cap \Omega} R_{\omega}^{\left(x^{i}\right)}\left(u\left\{\tilde{\omega}^{i}\right\} ; x, x^{i}\right) \phi_{k j}(x) d \Omega(x)\right],
\end{aligned}
$$

no sum in $i$. It is taken into account here that the domains $\omega^{i k}$ and $\Omega$ are open (do not include their boundaries $\partial \omega^{i k}$ and $\left.\partial \Omega\right)$. The approximate traction operators $T\left(u\left\{\tilde{\omega}^{i}\right\}\right)$ and $T^{i k}\left(u\left\{\tilde{\omega}^{i}\right\}\right)$, the localized parametrix $P_{\omega}^{i k}\left(u\left\{\tilde{\omega}^{i}\right\} ; x, x^{i}\right)$ and the localized remainder $R_{\omega}^{i k}\left(u\left\{\tilde{\omega}^{i}\right\} ; x, x^{i}\right)$ in (21) are expressed in terms of the set of unknowns $u\left\{\tilde{\omega}^{i}\right\}:=\left\{u\left(x^{j}\right), x^{j} \in \tilde{\omega}^{i}\right\}$. The expressions are obtained after substituting interpolation formulae (18), (19) for $u$ in the coefficient $a(u ; \cdot)$ in the definitions for $T(u)$, $T^{\left(x^{i}\right)}(u), P_{\omega}^{(y)}(u ; x, y)$ and $R_{\omega}^{(y)}(u ; x, y)$. The normal vector $n_{j}(x)$ for the operators $T(u), T^{\left(x^{i}\right)}(u)$ in the boundary integrals is taken outward to the corresponding domains, while for $a$ its limiting values are taken from inside of the domains. Integration is taken twice over the interfaces $\Omega \cap \partial \omega^{i k}$ between the adjoined domains $\omega^{i k}$. The right hand side components $\mathcal{F}_{\omega}\left(u\left\{\tilde{\omega}^{i}\right\}, x^{i}\right)$ in (20) are obtained after similar employing interpolation formulae (18), (19) for $u$ in (17).

Note that the term with $R_{\omega}^{i k}$ disappears in the last integral of (21) if the parametrix $P_{\omega}^{i k}\left(x, x^{i}\right)$ is given by (14). On the other hand, if the cut-off function $\chi\left(x, x^{i}\right)$ and its normal derivative are equal zero at $x$ on the boundary $\partial \omega\left(x^{i}\right)$, then the the third and fourth integrals along $\Omega \cap \partial \omega\left(x^{i}\right)$ disappear in $(21)$.

\subsection{Mesh-less discretization}

For a mesh-less discretization, one needs a method of local interpolation or approximation of a function along randomly distributed nodes $x^{i}$. We will suppose all the approximation nodes $x^{i}$ belong to $\bar{\Omega}$ and will use them also as collocation points for the LBDIDEs discretization. Let, as before, $J$ be the total number of nodes $x^{j}(i=1,2, \ldots, J)$. Let us consider a mesh-less method, for example, the moving 
least squares (MLS) (see e.g. [24]), that leads to the following approximation of a function $u(x)$

$$
u(x)=\sum_{x^{j} \in \omega_{0}(x)} \hat{u}\left(x^{j}\right) \Phi_{j}(x), x \in \Omega .
$$

Here $\Phi_{j}(x)$ are known smooth shape functions such that $\Phi_{j}(x)=0$ if $x^{j} \notin \omega_{0}(x), \omega_{0}(x)$ is a localization domain of the approximation formula, and $\hat{u}\left(x^{j}\right)$ are unknown values of an auxiliary function $\hat{u}(x)$ at the nodes $x^{j}$, that is, the so-called $\delta$-property is not assumed for approximation (22).

Let $\omega\left(x^{i}\right)$ be a localization domain around a node $x^{i}$. Then for any $x \in \bar{\omega}\left(x^{i}\right)$, the total approximation of $u(x)$ can be written in the following local form,

$$
u(x)=\sum_{x^{j} \in \tilde{\omega}^{i}} \hat{u}\left(x^{j}\right) \Phi_{j}(x), \nabla u(x)=\sum_{x^{j} \in \tilde{\omega}^{i}} \hat{u}\left(x^{j}\right) \nabla \Phi_{j}(x), x \in \bar{\omega}\left(x^{i}\right)
$$

where $\tilde{\omega}^{i}:=\cup_{x \in \bar{\omega}\left(x^{i}\right) \cap \bar{\Omega}} \omega_{0}(x)$ is a total localization domain, Fig. 3. Consequently, $\Phi_{j}(x)=\nabla \Phi_{j}(x)=0$ if $x \in \bar{\omega}\left(x^{i}\right)$ and $x^{j} \notin \tilde{\omega}^{i}$. Let $J_{\tilde{\omega}^{i}}$ be the number of nodes $x^{j} \in \tilde{\omega}^{i}$ and $\hat{u}\left\{\tilde{\omega}^{i}\right\}$ be the array of the function values $\hat{u}\left(x^{j}\right)$ at the node points $x^{j} \in \tilde{\omega}^{i}$. Since our approximation (23) for $u$ is smooth, its gradient approximation $\nabla u(x)$ is continuous, and can be directly applied in LBDIDE (16), unlike the mesh-based discretization.

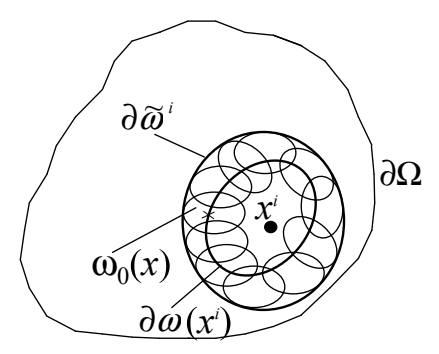

Figure 3: Localization domain $\omega\left(x^{i}\right)$ and a total localization domain $\tilde{\omega}^{i}$ associated with a collocation point $x^{i}$ of a body $\Omega$ for mesh-less discretizations

After substitution of approximation (23) in LBDIDE (16), we arrive at the following system of quasi-linear system of $J \times n$ algebraic equations with respect to $J \times n$ unknowns $\hat{u}_{m}\left(x^{j}\right), x^{j} \in \bar{\Omega}$, $m=1, \ldots, n$,

$$
\sum_{x^{j} \in \tilde{\omega}^{i}}\left[c\left(x^{i}\right) \Phi_{j}\left(x^{i}\right)+\hat{K}_{i j}\left(\hat{u}\left\{\tilde{\omega}^{i}\right\}\right)\right] \hat{u}\left(x^{j}\right)=\mathcal{F}_{\omega}\left(\hat{u}\left\{\tilde{\omega}^{i}\right\}, x^{i}\right), \quad x^{i} \in \bar{\Omega}, \quad \text { no sum in } i .
$$


For any $i, j$, the $n \times n$ tensor $\hat{K}_{i j}$ in $(24)$ is

$$
\begin{gathered}
\hat{K}_{i j}\left(\hat{u}\left\{\tilde{\omega}^{i}\right\}\right)=-\int_{\bar{\omega}\left(x^{i}\right) \cap \partial_{N} \Omega} \Phi_{j}(x)\left[T^{\left(x^{i}\right)}\left(\hat{u}\left\{\tilde{\omega}^{i}\right\}\right) P_{\omega}^{\left(x^{i}\right)}\left(\hat{u}\left\{\tilde{\omega}^{i}\right\} ; \cdot, x^{i}\right)\right](x) d \Gamma(x)+ \\
\int_{\bar{\omega}\left(x^{i}\right) \cap \partial_{D} \Omega} P_{\omega}^{\left(x^{i}\right)}\left(\hat{u}\left\{\tilde{\omega}^{i}\right\} ; x, x^{i}\right)\left[T\left(\hat{u}^{\prime}\left\{\tilde{\omega}^{i}\right\}\right) \Phi_{j}\right](x) d \Gamma(x)- \\
\int_{\Omega \cap \partial \omega\left(x^{i}\right)} \Phi_{j}(x)\left[T^{\left(x^{i}\right)}\left(\hat{u}\left\{\tilde{\omega}^{i}\right\}\right) P_{\omega}^{\left(x^{i}\right)}\left(\hat{u}\left\{\tilde{\omega}^{i}\right\} ; \cdot, x^{i}\right)\right](x) d \Gamma(x)+ \\
\int_{\Omega \cap \partial \omega\left(x^{i}\right)} P_{\omega}^{\left(x^{i}\right)}\left(\hat{u}\left\{\tilde{\omega}^{i}\right\} ; x, x^{i}\right)\left[T\left(\hat{u}\left\{\tilde{\omega}^{i}\right\}\right) \Phi_{j}\right](x) d \Gamma(x)- \\
\int_{\omega\left(x^{i}\right) \cap \Omega}\left[\nabla^{(x)} P_{\omega}^{\left(x^{i}\right)}\left(\hat{u}\left\{\tilde{\omega}^{i}\right\} ; x, x^{i}\right)\right] \tilde{a}\left(\hat{u}\left\{\tilde{\omega}^{i}\right\} ; x, x^{i}\right) \nabla \Phi_{j}(x) d \Omega(x)+ \\
\int_{\omega\left(x^{i}\right) \cap \Omega} R_{\omega}^{\left(x^{i}\right)}\left(\hat{u}\left\{\tilde{\omega}^{i}\right\} ; x, x^{i}\right) \Phi_{j}(x) d \Omega(x),
\end{gathered}
$$

with the shape functions $\Phi_{j}$ from (23). Expressions for $T\left(\hat{u}\left\{\tilde{\omega}^{i}\right\}\right), P_{\omega}\left(\hat{u}\left\{\tilde{\omega}^{i}\right\} ; x, x^{i}\right)$ and $R_{\omega}\left(\hat{u}\left\{\tilde{\omega}^{i}\right\} ; x, x^{i}\right)$ in terms of the set of unknowns $\hat{u}\left\{\tilde{\omega}^{i}\right\}:=\left\{\hat{u}\left(x^{j}\right), x^{j} \in \tilde{\omega}^{i}\right\}$ are obtained after substituting interpolation formulae (23) for $u$ in the coefficient $a(u ; \cdot)$ in the definitions for $T(u), P_{\omega}(u ; x, y)$ and $R_{\omega}(u ; x, y)$. The right hand side components $\mathcal{F}_{\omega}\left(\hat{u}\left\{\tilde{\omega}^{i}\right\}, x^{i}\right)$ are obtained after similar employing interpolation formulae (23), for $u$ in (17).

\section{Concluding remarks}

The parametrix localization by multiplication by a cut-off function with a local support allows to reduce a BVP of the non-linear elasticity to a two-operator direct localized quasi-linear boundarydomain integro-differential equation of the second kind. The equation includes at most the first derivative of the unknown solution, weakly singular integrals over the domain, and at most Cauchytype singular integrals over the boundary. The second kind structure of the nonlinear LBDIDE and of the corresponding mesh-based discrete system look very promising for constructing simple and fast converging iteration algorithms.

From the definitions in both mesh based and mesh-less discretization methods, we have $\phi_{k j}(x)=$ $\nabla \phi_{k j}(x)=\Phi_{j}(x)=\nabla \Phi_{j}(x)=\left[T(u) \phi_{k j}\right](x)=\left[T^{(y)}(u) \phi_{k j}\right](x)=\left[T(u) \Phi_{j}\right](x)=\left[T^{(y)}(u) \Phi_{j}\right](x)=0$ if $x \in \bar{\omega}\left(x^{i}\right)$ but $x^{j} \notin \tilde{\omega}^{i}$. Consequently $K_{i j}=0$ and $\hat{K}_{i j}=0$ if $x^{j} \notin \tilde{\omega}^{i}$, and moreover, $K_{i j}$ and $\hat{K}_{i j}$ depend only on $u\left\{\tilde{\omega}^{i}\right\}$ or $\hat{u}\left\{\tilde{\omega}^{i}\right\}$, respectively. Thus, each equation in (20) and (24) has not more than $J_{\tilde{\omega}^{i}} \times n \ll J \times n$ non-zero entries, i.e. the systems are sparse. The number $J_{\tilde{\omega}^{i}} \times n$ of nonzero entries is practically independent of the mesh refinement but depends on the domain element types in the mesh-based discretization. The similar effect takes place also in the mesh-less discretization if the global localization domain $\tilde{\omega}\left(x^{i}\right)$ shrinks with the refinement of the nodes distribution.

Deriving two-operator BDIDE (13), we employed the auxiliary linear constant-coefficient operators $L^{(y)}$ and $T^{(y)}$, given by (5), (6) in terms of the secant "frozen" elastic tensor $a_{i j k l}(\nabla u(y), u(y), y)$. 
Another possible option would be to use for this purpose the initial linear constant-coefficient operators $L^{(0 y)}$ and $T^{(0 y)}$ associated with the initial "frozen" elastic tensor $a_{i j k l}(0,0, y)$. The resulting BDIDE would then be given by the same equation (13) after replacing there $a_{i j k l}(\nabla u(y), u(y), y)$ by $a_{i j k l}(0,0, y)$ everywhere, including the operators $L^{(y)}$ and $T^{(y)}$, and fundamental solution $F^{(y)}$. The localization and discretization procedures described for the secant-coefficient LBDIDE will be equally applicable also to this initial-coefficient LBDIDE. However, the difference tensor $\tilde{a}_{i j k l}^{0}(u ; x, y):=\left[a_{i j k l}(\nabla u(x), u(x), x)-\right.$ $\left.a_{i j k l}(0,0, y)\right]$ will not tend to zero as $x \rightarrow y$, unlike its counterpart $\tilde{a}_{i j k l}(u ; x, y)$ given by (8), which can influence properties of the integral equation and its discrete counterparts.

Investigation of the equivalence of the BDIDEs to the original BVPs, solvability, uniqueness of solution, and the iteration algorithm convergence, including analysis of spectral properties of the corresponding linear BDIDEs, needs to be done for constructing robust numerical methods based on this information (c.f. [25]), and for an optimal choice of the cut-off functions, localization domains and node points.

\section{References}

[1] Brebbia CA, Telles JCF, Wrobel LC. Boundary Element Techniques, Berlin: Springer; 1984.

[2] Balaš J, Sladek J, Sladek V. Stress Analysis by Boundary Element Methods. Amsterdam-OxfordNew York-Tokyo: Elsevier; 1989.

[3] Banerjee PK. The Boundary Element Methods in Engineering, London: McGraw-Hill; 1994.

[4] Aliabadi MH, The Boundary Element Method, Vol. 2, Applications in Solids and Structures, Chichester: Wiley; 2002.

[5] Mikhailov SE. Localized boundary-domain integral formulations for problems with variable coefficients. Engng Anal Bound Elem 2002;26:681-690.

[6] Mikhailov SE, Nakhova IS. Numerical solution of a Neumann problem with variable coefficients by the localized boundary-domain integral equation method. In: Amini S. editor. Fourth UK Conference on Boundary Integral Methods, Salford University; 2003. p. 175-184.

[7] Mikhailov SE, Nakhova IS. Mesh-based numerical implementation of the localized boundarydomain integral equation method to a variable-coefficient Neumann problem. J Engng Math 2005;51:251-259.

[8] Sladek V, Sladek J. A new formulation for solution of boundary value problems using domain-type approximations and local integral equations. Electronic J. Boundary Elements 2003;1:132-153. 
[9] Sladek J, Sladek V, Zhang Ch. Local integro-differential equations with domain elements for the numerical solution of partial differential equations with variable coefficients. J Engng Math $2005 ; 51: 261282$.

[10] Zhu T, Zhang J-D, Atluri SN. A local boundary integral equation (LBIE) method in computational mechanics, and a meshless discretization approach. Comput Mech 1998;21:223-235.

[11] Zhu T, Zhang J-D, Atluri SN. A meshless numerical method based on the local boundary integral equation (LBIE) to solve linear and non-linear boundary value problems. Engng Anal Bound Elem 1999;23:375-389.

[12] Sladek J, Sladek V, Zhang Ch. A local BIEM for analysis of transient heat conduction with nonlinear source terms in FGMs. Engng Anal Bound Elem 2004;28:1-11.

[13] Sladek J, Sladek V, Atluri SN. Local boundary integral equation (LBIE) method for solving problems of elasticity with nonhomogeneous material properties. Comput Mech 2000;24:456-462.

[14] Sladek J, Sladek V, Krivacek J, Zhang Ch. Local BIEM for transient heat conduction analysis in 3-D axisymmetric functionally graded solids. Comput Mech 2003;32:169176.

[15] Mikhailov SE. About localized boundary-domain integro-differential formulations for a quasilinear problem with variable coefficients. In: Constanda C, Ahues M, Largillier A, editors. Integral Methods in Science and Engineering: Analytic and Numerical Techniques, Birkhäuser; 2004. p. 139-144.

[16] Mikhailov SE. Some localized boundary- domain integro-differential formulations for quasi-linear problems with variable coefficients. In: Gallego R, Aliabadi MH, editors. Advances in Boundary Element Techniques IV, Queen Mary, Univ. of London; 2003, p. 289-294.

[17] Mikhailov SE. Localized direct boundary-domain integro-differential formulations for some nonlinear problems with variable coefficients. J Engng Math 2005;51:283-302.

[18] Mikhailov SE. Localized boundary-domain integro-differential formulation for physically nonlinear elasticity of inhomogeneous body. In: Leitao VMA, Aliabadi MH, editors Advances in Boundary Element Techniques V. EC Ltd., UK, ISBN 09547783-0-8; 2004. p. 31-38.

[19] Mikhailov SE. Localized boundary-domain integro-differential formulation for incremental elastoplasticity of inhomogeneous body. In: Yao ZH, Yuan MW, Zhong WX, editors. Computational Mechanics, WCCM VI in conjunction with APCOM'04, Sept. 5-10, 2004, Beijing, China, Tsinghua University Press \& Springer-Verlag, ISBN 7-89494-512-9; 2004. 9p.

[20] Synge JL. Hypercircle in Mathematical Physics. Cambridge Univ Press; 1957.

[21] Vogel SM, Rizzo FJ. An integral equation formulation of three-dimensional anisotropic boundary value problems. J Elast 1973;3: 203-216. 
[22] Wilson RB, Cruse TA. Efficient implementation of anisotropic three-dimensional boundary integral equation stress analysis. Intern J Numer Methods Engng1978;12:1383-1397.

[23] Miranda C. Partial Differential Equations of Elliptic Type, Berlin: Springer; 1970.

[24] Belytschko T, Krongauz Y, Organ D, Flemming M, Krysl P. Meshless methods: An overview and recent developments. Comput Methods Appl Mech Engng 1996;139:4-47.

[25] Mikhailov SE. Finite-dimensional perturbations of linear operators and some applications to boundary integral equations. Engng Anal Bound Elem 1999;23:805-813. 